\title{
Faster Deterministic Fully-Dynamic Graph Connectivity
}

\author{
Christian Wulff-Nilsen *
}

\begin{abstract}
We give new deterministic bounds for fully-dynamic graph connectivity. Our data structure supports updates (edge insertions/deletions) in $O\left(\log ^{2} n / \log \log n\right)$ amortized time and connectivity queries in $O(\log n / \log \log n)$ worst-case time, where $n$ is the number of vertices of the graph. This improves the deterministic data structures of Holm, de Lichtenberg, and Thorup (STOC 1998, J.ACM 2001) and Thorup (STOC 2000) which both have $O\left(\log ^{2} n\right)$ amortized update time and $O(\log n / \log \log n)$ worst-case query time. Our model of computation is the same as that of Thorup, i.e., a pointer machine with standard $A C^{0}$ instructions.
\end{abstract}

\footnotetext{
*Department of Computer Science, University of Copenhagen, koolooz@diku.dk, http://www.diku.dk/ koolooz/.
} 


\section{Introduction}

The dynamic graph connectivity problem is perhaps the most fundamental dynamic graph problem and has received considerable attention from the algorithms community for decades. The goal is to build an efficient data structure that supports one or more of the following operations in a dynamic graph $G$ :

- connected $(u, v)$ : determines whether vertices $u$ and $v$ are connected in $G$,

- $\operatorname{insert}(u, v)$ : inserts edge $(u, v)$ in $G$,

- delete $(u, v)$ : deletes edge $(u, v)$ from $G$.

The fully-dynamic graph connectivity problem supports all three operations, whereas the simpler decremental and incremental variants do not support insert and delete, respectively. In the following, we refer to both insert and delete as update operations.

The first non-trivial data structure for fully-dynamic graph connectivity is due to Frederickson [2] who showed how to support updates in $O(\sqrt{m})$ time and connectivity queries in $O(1)$ time, where $m$ is the number of edges of the graph. Using a general sparsification technique, Eppstein, Galil, Italiano, and Nissenzweig [1] improved update time to $O(\sqrt{n})$, where $n$ is the number of vertices. Both of these data structures are deterministic and the time bounds are worst-case.

Henzinger and King [3] significantly improved update time to $O\left(\log ^{3} n\right)$ with only a small increase in query time to $O(\log n / \log \log n)$. However, their bounds are randomized expected and update time is amortized. Using a clever sampling technique, Henzinger and Thorup [4] shaved off a factor of $\log n$ in the update time. A simple and elegant deterministic data structure with the same bounds as in [4] was given by Holm, de Lichtenberg, and Thorup [5]. Its space requirement was later improved to linear by Thorup [10] who also gave a randomized data structure with a faster update time of $O\left(\log n(\log \log n)^{3}\right)$ and marginally slower query time of $O(\log n / \log \log \log n)$.

A general cell-prove lower bound of $\Omega(\log n)$ for fully-dynamic graph connectivity was provided by Pătraşcu and Demaine [6]. Hence, the data structures above are near-optimal.

As shown by Tarjan [8], incremental connectivity is the union-find problem which can be solved in $O(\alpha(m, n))$ time over $m$ updates. Thorup [9] gave an $O(\log n)$ bound for decremental connectivity if the initial graph has $\Omega\left(n \log ^{5} n\right)$ edges. He also gave an $O(1)$ bound if the initial graph is dense.

Our contribution is a deterministic data structure that improves the update time of the deterministic data structures in [5, 10, by a factor of $\log \log n$. We use several ingredients of Thorup [10], including his structural forest (which we refer to as a cluster forest) as well as lazy local trees and shortcuts both of which we modify to suit our needs. We also introduce an additional system of shortcuts that allows us to more quickly walk up trees of the cluster forest. Together, these changes and additions give an order $\log \log n$ speed-up in the update time.

Table 1 summarizes the results for fully-dynamic graph connectivity.

Our paper is organized as follows. In Section 2, we introduce basic definitions and notation. Section 3 gives a simple data structure with $O\left(\log ^{2} n\right)$ update time and $O(\log n)$ query time. It is essentially the deterministic data structure of Thorup [10] but a slightly more minimalistic variant that does not need to maintain spanning trees of connected components. In Section 4 , 


\begin{tabular}{|c|c|c|c|}
\hline Update time & Query time & Type & Reference \\
\hline$O(\sqrt{m})$ & $O(1)$ & deterministic; worst-case time & {$[2]$} \\
\hline$O(\sqrt{n})$ & $O(1)$ & deterministic; worst-case time & {$[1]$} \\
\hline$O\left(\log ^{3} n\right)$ & $O(\log n / \log \log n)$ & randomized; amortized update time & {$[3]$} \\
\hline$O\left(\log ^{2} n\right)$ & $O(\log n / \log \log n)$ & randomized; amortized update time & {$[4]$} \\
\hline$O\left(\log ^{2} n\right)$ & $O(\log n / \log \log n)$ & deterministic; amortized update time & {$[5$, 10] } \\
\hline$O\left(\log n(\log \log n)^{3}\right)$ & $O(\log n / \log \log \log n)$ & randomized; amortized update time & {$[10$} \\
\hline$O\left(\log ^{2} n / \log \log n\right)$ & $O(\log n / \log \log n)$ & deterministic; amortized update time & This paper \\
\hline
\end{tabular}

Table 1: Performance of data structures for fully-dynamic graph connectivity.

we add two systems of shortcuts to our data structure that together improve both update and query time by a factor of $\log \log n$. The simplification given in Section 3 is not important in order to get our improvement in Section 4: indeed, our result can easily be extended to maintain a spanning forest. However, we believe that our approach gives a slightly cleaner analysis and it should give a small improvement in practice. For instance, Thorup's data structure needs to maintain two types of bitmaps for edges, one for tree edges and one for non-tree edges whereas our data structure only needs to maintain one type; see Section 3.5 for details. Finally, we give some concluding remarks in Section 5 .

\section{Preliminaries}

Let log denote the base 2 logarithm. We assume the same model of computation as in [10], i.e., a pointer machine with words (bitmaps) containing at least $\lfloor\log n\rfloor+1$ bits and with the following standard $A C^{0}$ instructions: addition, subtraction, bitwise 'and', 'or', and 'not', and bit shifts. Our data structure also needs to perform division $x / y$ and multiplication $x y$ which are not $A C^{0}$ instructions. To handle this, we assume that $y$ is a power of 2 so that a bit shift operation can be used instead; we can always round $y$ to the nearest such value and the constant multiplicative error introduced will not affect correctness or running time. For a bitmap $m$, we denote by $m[i]$ the $i$ th bit of $m, i \geq 0$.

We let $G$ denote the input graph and it is assumed to contain no edges initially. To distinguish between vertices of $G$ and other vertices (such as those in trees of our data structure), we refer to the latter as nodes. For a path $P$ and nodes $a, b \in P, P[a, b]$ is the subpath of $P$ between $a$ and $b$. We abbreviate balanced binary search tree as BBST and depth-first search as DFS.

\section{A Simple Data Structure}

We first give a simplified version of our data structure which is no better than the deterministic data structures in [5] and [10]. In fact, it has a slower query time of $O(\log n)$. In Section 4 , we shall speed up both query and update time by a factor of $\log \log n$. 


\subsection{The cluster forest}

As in [10], we assign to each edge $e$ of $G$ a level $\ell(e)$ between 0 and $\ell_{\max }=\lfloor\log n\rfloor$ and for $0 \leq i \leq l_{\max }$, we denote by $G_{i}$ the subgraph of $G$ induced by edges $e$ with $\ell(e) \geq i$. We refer to the connected components of $G_{i}$ as level $i$ clusters or just clusters. The following invariant will be maintained by our data structure:

Invariant: For each $i$, any level $i$ cluster spans at most $\left\lfloor n / 2^{i}\right\rfloor$ vertices.

The cluster forest of $G$ is a forest $\mathcal{C}$ of rooted trees where each node $u$ corresponds to a cluster $C(u)$. The level $\ell(u)$ of $u$ is its depth in $\mathcal{C}$ (between 0 and $\ell_{\max }$ ) and a level $i$ node corresponds to a level $i$ cluster. In particular, roots of $\mathcal{C}$ correspond to level 0 clusters and hence to the connected components of $G_{0}=G$. By the invariant, each leaf of $\mathcal{C}$ corresponds to a vertex of $G$; we often identify the two and our data structure keeps bidirected pointers between them. A node $u$ at a level $i<\ell_{\max }$ has as children the level $(i+1)$ nodes $v$ such that $C(v) \subseteq C(u)$.

Our data structure will maintain, for each node $u$ of $\mathcal{C}$, an integer $n(u)$ denoting the number of leaves in the subtree of $\mathcal{C}$ rooted at $u$. In other words, $n(u)$ is the number of vertices of $G$ spanned by $C(u)$. This completes the description of the cluster forest.

Given $\mathcal{C}$, we can determine whether two vertices $u$ and $v$ are connected in $G$ in $O(\log n)$ time as follows. Traverse paths from $u$ and $v$ to roots $r_{u}$ and $r_{v}$ of trees of $\mathcal{C}$ containing $u$ and $v$, respectively. Then $u$ and $v$ are connected iff $r_{u}=r_{v}$.

\subsection{Handling insertions}

We need to maintain $\mathcal{C}$ as $G$ changes. First we describe how to update $\mathcal{C}$ after an operation insert $(u, v)$. We initialize $\ell(u, v) \leftarrow 0$. Letting $r_{u}$ and $r_{v}$ be defined as above, if $r_{u}=r_{v}$, no update of $\mathcal{C}$ is required since $u$ and $v$ were already connected in $G=G_{0}$. If $r_{u} \neq r_{v}$, we update $\mathcal{C}$ by merging $r_{u}$ and $r_{v}$ into $r_{u}$, meaning that $r_{u}$ inherits the children of $r_{v}, n\left(r_{u}\right)$ is increased by $n\left(r_{v}\right)$, and $r_{v}$ is deleted. This update corresponds to merging $C\left(r_{u}\right)$ and $C\left(r_{v}\right)$. Thus $\mathcal{C}$ is correctly updated and the invariant still holds.

\subsection{Handling deletions}

Now consider the update delete $(u, v)$. Let $i=\ell(u, v)$ and let $C_{u}$ and $C_{v}$ be the level $(i+1)$ clusters containing $u$ and $v$, respectively. Assume that $C_{u} \neq C_{v}$ since otherwise, the connectivity in $G_{i}$ is not affected (there is a $u v$-path in $G_{i+1} \subset G_{i}$ connecting $u$ and $v$ ). Let $M_{i}$ be the multigraph with level $(i+1)$ clusters as vertices and with level $i$-edges of $G$ as edges (so an edge of $M_{i}$ connects two vertices if that edge connects the corresponding level $(i+1)$ clusters in $\left.G_{i}\right)$. Our algorithm will not actually keep $M_{i}$ but it will help to simplify the description in this subsection.

We now execute two standard search procedures (say, DFS) in $M_{i}$, one, $P_{u}$, starting from vertex $C_{u}$ and another, $P_{v}$, starting from $C_{v}$. The two procedures are executed in "parallel" by alternating between the two, i.e., one unit of time is spent on $P_{u}$, then one unit on $P_{v}$, then one unit on $P_{u}$, and so on. We terminate both procedures as soon as we are in one of the following two cases (which must happen at some point):

1. a vertex of $M_{i}$ is explored by both procedures,

2. one of the procedures has no more edges to explore and we are not in case 1 . 
In the following, we show how to deal with these two cases.

Case 1: Let $C_{u v}$ be the vertex (level $(i+1)$ cluster) of $M_{i}$ explored by both procedures. Assume w.l.o.g. that $P_{v}$ was the last to explore $C_{u v}$. Let $\mathcal{C}_{u}$ be the set of level $(i+1)$ clusters explored by $P_{u}$ and let $\mathcal{C}_{v}$ be the set of level $(i+1)$ clusters explored by $P_{v}$, excluding $C_{u v}$. If we let $n_{u}=\sum_{C \in \mathcal{C}_{u}} n(C)$ and $n_{v}=\sum_{C \in \mathcal{C}_{v}} n(C)$ then since $\mathcal{C}_{u} \cap \mathcal{C}_{v}=\emptyset$, we have $n_{u}+n_{v} \leq\left\lfloor n / 2^{i}\right\rfloor$ by our invariant and thus $\min \left\{n_{u}, n_{v}\right\} \leq\left\lfloor n / 2^{i+1}\right\rfloor$.

Assume first that $n_{u} \leq n_{v}$. Then we can increase the level of every edge explored by $P_{u}$ from $i$ to $i+1$ without violating the invariant. To see this, note that the level updates correspond to merging clusters of $\mathcal{C}_{u}$ into one level $(i+1)$ cluster spanning $n_{u} \leq\left\lfloor n / 2^{i+1}\right\rfloor$ vertices. The idea is that the search performed by $P_{u}$ is paid for by these level increases. As $P_{v}$ spent the same amount of time as $P_{u}$ (up to an additive $O(1)$ term), the level increases also pay for the search by $P_{v}$.

We need to update cluster forest $\mathcal{C}$ accordingly. When increasing the level of an edge $e$ from $i$ to $i+1$, we identify the level $(i+1)$-ancestors $a$ and $b$ of the endpoints of $e$. Clusters $C(a)$ and $C(b)$ need to be merged and we do this by merging $a$ and $b$ into $b$ and updating $n(a) \leftarrow n(a)+n(b)$. As we will see later, this update can also be paid for by the level increase of $e$. Note that the procedures have found a replacement path in $G_{i}$ for deleted edge $(u, v)$ so no further updates are required in $\mathcal{C}$, and we terminate.

We assumed above that $n_{u} \leq n_{v}$. We do exactly the same for clusters in $\mathcal{C}_{v}$ when $n_{v} \leq n_{u}$ except that we do not increase the level of the last edge explored by $P_{v}$ as it connects to a cluster in $\mathcal{C}_{u}$. If this was the only edge explored, there are no edges to pay for it but in this case we have found a replacement path for edge $(u, v)$ and the delete $(u, v)$-operation can pay.

Case 2: Now assume that one of the procedures, say $P_{u}$, explores all edges in the connected component of $M_{i}$ containing $C_{u}$ and that we are not in case 1 . Let us assume that $n_{u} \leq$ $\left\lfloor n / 2^{i+1}\right\rfloor$; if not, we fully explore the connected component of $M_{i}$ containing $C_{v}$ and update $n_{v}$ which will be at most $\left\lfloor n / 2^{i+1}\right\rfloor$ by our invariant; the description below then applies if we swap the roles of $u$ and $v$.

We can conclude that no replacement path for $(u, v)$ exists in $G_{i}$. All edges explored by $P_{u}$ have their level increased to $i+1$ and we update $\mathcal{C}$ accordingly by merging all level $(i+1)$ nodes explored by $P_{u}$ into one, $w$, and setting $n(w)$ to the sum of $n\left(w^{\prime}\right)$ for nodes $w^{\prime}$ explored by $P_{u}$. These level increases pay for the two searches. Since $C_{u}$ and the component of $M_{i}$ containing $C_{v}$ are no longer connected in $G_{i}$, we further update $\mathcal{C}$ as follows: let $p$ be the parent of $w$ in $\mathcal{C}$. We remove $w$ as a child of $p$, decrease $n(p)$ by $n(w)$, add $w$ as a child of a new level $i$ node $p^{\prime}$, set $n\left(p^{\prime}\right)=n(w)$, and add $p^{\prime}$ as a child of the parent of $p$. This correctly updates $\mathcal{C}$ and the invariant is maintained.

If $i>0$, it may still be possible to reconnect $u$ and $v$ in $G_{j}$ for some $j<i$. We thus execute the above algorithm recursively with $i \leftarrow i-1, C_{u} \leftarrow C\left(p^{\prime}\right)$, and $C_{v} \leftarrow C(p)$. Should we end up in case 2 with $i=0$, no replacement path in $G$ between $u$ and $v$ could be found. Then $p^{\prime}$ becomes a new root of $\mathcal{C}$ and we terminate.

\subsection{Local trees}

We now extend our data structure to allow the search procedures to explore edges and vertices of multigraph $M_{i}$ in a more efficient way. 
First we shall convert $\mathcal{C}$ into a forest of binary trees by adding local trees as in [10]. Let $u$ be a non-leaf node of $\mathcal{C}$. We form a binary tree $L(u)$ with $u$ as root and with the children of $u$ as leaves as follows. First assign a $\operatorname{rank} \operatorname{rank}(v) \leftarrow\lfloor\log n(v)\rfloor$ to each child $v$ of $u$. Initially, each such $v$ is regarded as a tree consisting just of $v$. While there are trees $T$ and $T^{\prime}$ whose roots $r$ and $r^{\prime}$ have the same rank, we pair them by attaching $r$ and $r^{\prime}$ to a new root $r^{\prime \prime}$ with $\operatorname{rank}\left(r^{\prime \prime}\right) \leftarrow \operatorname{rank}(r)+1$. We end up with at most $\log n$ trees $T_{1}, \ldots, T_{k}$, called rank trees, whose roots $r_{1}, \ldots, r_{k}$ have pairwise distinct ranks: $\operatorname{rank}\left(r_{1}\right)>\operatorname{rank}\left(r_{2}\right)>\cdots>\operatorname{rank}\left(r_{k}\right)$. We connect the rank trees into a single local tree $L(u)$ rooted at $u$ by adding a rank path $v_{1} v_{2} \ldots v_{k-1}$ down from $v_{1}=u$ and connecting $r_{i}$ as a child to $v_{i}$ for $i=1, \ldots, k-1$ and $r_{k}$ as a child to $v_{k-1}$. We $\operatorname{define} \operatorname{rank}(u) \leftarrow\lfloor\log n(u)\rfloor$.

The edges in $\mathcal{C}$ from $u$ to its children are replaced by local tree $L(u)$; let $\mathcal{C}_{L}$ be $\mathcal{C}$ after all local trees have been added. As shown by Thorup, for a child $v$ of a node $u$ in $\mathcal{C}$, the depth of $v$ in $L(u)$ is at most $\log (n(u) / n(v))+1$. Since any leaf of $\mathcal{C}$ has depth at most $\ell_{\max }=\lfloor\log n\rfloor$, a telescoping sums argument implies that any leaf of $\mathcal{C}_{L}$ has depth $O(\log n)$.

Refer to nodes of $\mathcal{C}_{L}$ that are also nodes of $\mathcal{C}$ as $\mathcal{C}$-nodes. Our data structure will maintain $\mathcal{C}_{L}$ as well as $n(u)$ for each $\mathcal{C}$-node $u$ and $\operatorname{rank}(v)$ for each node $v \in \mathcal{C}_{L}$.

\subsection{Searching for edges}

We shall use $\mathcal{C}_{L}$ to search for edges in $M_{i}$. To facilitate this, we associate a bitmap edge $(u)$ with each node $u$ of $\mathcal{C}_{L}$ where edge $(u)[i]=1$ iff a level $i$-edge is incident to a leaf of the subtree of $\mathcal{C}_{L}$ rooted at $u \mathbb{1}$

We can use these bitmaps to search for the edges of $M_{i}$. Consider one of the search procedures, say $P_{u}$, described above. At any point in the search, a set of vertices of $M_{i}$ have been explored and these correspond to level $(i+1)$ nodes in $\mathcal{C}_{L}$ that we mark as explored. With the bitmaps, we identify unexplored descendant leaves of marked nodes in $\mathcal{C}_{L}$ that are incident to level $i$-edges and hence to edges of $M_{i}$ that should be explored by $P_{u}$. At each leaf, we have all incident edges grouped according to their level. A BBST is kept which allows us to get down to a particular group in $O\left(\log \ell_{\max }\right)=O(\log \log n)$ time. When a level $i$-edge $(a, b)$ is explored in the direction from $a$ to $b$, we determine the endpoint in $M_{i}$ corresponding to $b$ by moving up from leaf $b$ to the ancestor level $(i+1)$ node in $\mathcal{C}_{L}$. Finally, we mark this level $(i+1)$ node as explored. Since $\mathcal{C}_{L}$ has $O(\log n)$ height, we can execute $P_{u}$ in $O(\log n)$ time per edge explored.

\subsection{Maintaining $\mathcal{C}_{L}$}

We now describe how to maintain $\mathcal{C}_{L}$ as $\mathcal{C}$ is updated. Let us consider the update in $\mathcal{C}$ of merging nodes $u$ and $v$ into $u$. In $\mathcal{C}_{L}$, this is done by first removing the rank paths in $L(u)$ and $L(v)$, leaving at most $\log n$ rank trees of distinct ranks for each of the nodes $u$ and $v$. We may assume that rank trees are kept in two lists sorted by the ranks of their roots and we merge the two lists into one and start pairing up trees whose roots have the same rank, in the same way as above. We connect their roots with a new rank path, thereby creating the new $L(u)$ and we identify its root with $u$. Total time for a merge is $O(\log n)$.

We also need to update $\mathcal{C}_{L}$ when a child $b$ in $\mathcal{C}$ is added to or removed from a node $a$ (we need this when failing to find a replacement path at some level). If $b$ is to be added, we can

\footnotetext{
${ }^{1}$ Thorup's data structure needs two bitmaps in order to distinguish between tree edges and non-tree edges whereas we only need one; edge $(u)$ can be regarded as the bitwise 'or' of his two bitmaps.
} 
regard it as a trivial rank tree that should be added to $L(a)$. This can be done in $O(\log n)$ time using the same approach as for merging. If $b$ is to be removed, we first remove the rank path of $L(a)$ and identify the rank tree $T_{b}$ containing $b$. We delete the path from $b$ to the root of $T_{b}$, thereby partitioning this rank tree into $O(\log n)$ smaller rank trees, sorted by ranks. We pair up rank trees as described above and add a new rank path to form the updated $L(a)$. All of this can be done in $O(\log n)$ time.

\subsection{Maintaining bitmaps}

Finally, we need to update integers $n(u)$ for $\mathcal{C}$-nodes $u$ as well as the edge-bitmaps. The former is done exactly as in Sections 3.2 and 3.3 so let us focus on the bitmaps. If a level $i$-edge $e$ is removed, we do the following for each of its endpoints $a$. In the leaf $a$ of $\mathcal{C}_{L}$, we check in $O(\log \log n)$ time if $e$ was the only level $i$-edge incident to $a$. If so, we set edge $(a)[i] \leftarrow 0$ and we move up $\mathcal{C}_{L}$, updating the bitmap of the current node as the bitwise 'or' of its children. Since $\mathcal{C}_{L}$ has $O(\log n)$ height, only $O(\log n)$ bitmaps need to be updated. Similarly, if $e$ is added, we set edge $(a)[i] \leftarrow 1$ and update bitmaps for ancestors in the same way. For nodes of $\mathcal{C}_{L}$ whose children change, we also update their bitmaps bottom up by taking the bitwise 'or' of their children. Only $O(\log n)$ nodes are affected in $\mathcal{C}_{L}$ after an update in $\mathcal{C}$ so total time is $O(\log n)$.

This completes the description of the first version of our data structure. Correctness follows since the data structure is a simple variation of that of Thorup where spanning trees of clusters are not kept; rather, our search procedures certify that a spanning tree exists for an explored component and this suffices to maintain the cluster forest. From the analysis above, our data structure handles updates in $O\left(\log ^{2} n\right)$ amortized time and queries in $O(\log n)$ time. In the next section, we speed up both bounds by a factor of $\log \log n$.

\section{An Improved Data Structure}

In this section, we give our improved data structure. Before going into details, let us highlight the main differences between this structure and that of the previous section. One ingredient is to add shortcuts to $\mathcal{C}_{L}$. Each shortcut skips $O(\log \log n)$ nodes and this will allow our search procedures to walk up trees of $\mathcal{C}_{L}$ in $O(\log n / \log \log n)$ time per traversal when identifying visited nodes of a multigraph $M_{i}$. Adding these shortcuts essentially corresponds to turning the forest $\mathcal{C}_{L}$ of binary trees into one consisting of trees with a branching factor of order $\log n$, and reducing the height of the trees to order $\log n / \log \log n$. Furthermore, we will modify $\mathcal{C}_{L}$ by using lazy local trees similar to those of Thorup [10] instead of the local trees presented in the previous section. This is done to maintain $\mathcal{C}_{L}$ more efficiently during changes. Unfortunately, Thorup's lazy local trees increase the height of $\mathcal{C}_{L}$ to $O(\log n \log \log n)$ so our shortcuts will not give any speed-up over the data structure in the previous section. Instead, we shall use a slightly more complicated type of lazy local tree which has the properties we need while keeping the height of $\mathcal{C}_{L}$ bounded by $O(\log n)$. The idea is to partition the children of each node of $\mathcal{C}$ into so called heavy children and light children and construct the lazy local tree only for the light children and the local tree of the previous section for the heavy children. Balancing this in the right way will ensure a logarithmic depth of trees while still getting the speed-up from lazy local trees. Finally, we will need another system of shortcuts for quickly identifying edges to be explored by the search procedures; Thorup uses a similar system but

it does not fit into our framework as our lazy local trees are different from his. As shown in 


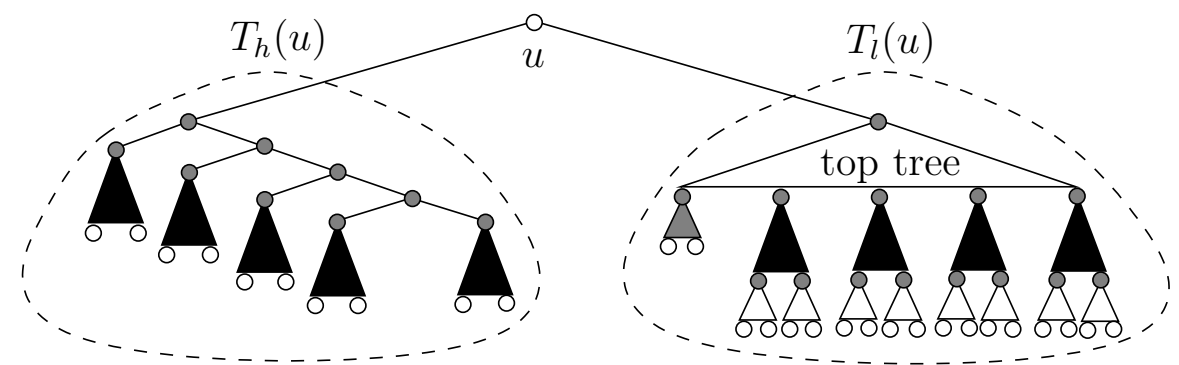

Figure 1: Lazy local tree $L(u)$. Rank trees are black, top and bottom trees are white, the buffer tree is grey, nodes of $L(u) \cap \mathcal{C}$ are white, and nodes of $L(u) \backslash \mathcal{C}$ are grey.

Lemma 6 in Section 4.7, with these shortcuts, the search procedures can visit edges in only $O(\log n / \log \log n)$ time per edge plus an additive $O(\log n)$ if we are in case 1 in Section 3.3 , note that the latter can be paid for by the delete operation since a replacement path for the deleted edge has been found. In Section 4.6, we define these shortcuts and in Section 4.7, we give an algorithm that uses these shortcuts to explore level $i$-edges; we refer to it as a level $i$-iterator or just iterator.

\subsection{Lazy local trees}

Thorup [10] introduced lazy local trees and showed how they can be maintained more efficiently than the local trees in Section 3.4. Let $u$ be a non-leaf node of $\mathcal{C}$ and let $L$ be the set of children of $u$. To form the lazy local tree of $u, L$ is divided into groups each of size at most $2(\log n)^{\alpha}$, where $\alpha$ is a constant that we may pick as large as we like. The nodes in each group are kept in a BBST ordered by $n(v)$-values. One of these trees is the buffer tree while the others are the bottom trees. The root of a bottom tree has rank equal to the maximum rank of its leaves. These bottom trees are paired up to form at most $\log n$ rank trees, as described in Section 3.4. The roots of the rank trees together with the root of the buffer tree are leaves of a BBST called the top tree where leaves are ordered according to rank (and the root of the buffer tree is regarded as, say, the smallest element). Together, these trees form the lazy local tree of $u$ which is rooted at the root of the top tree. Note that bottom, buffer, and top trees have polylogarithmic size only. It is ensured by the data structure of Thorup that for each bottom tree $B$, new leaves are never added to $B$ and ranks of leaves in $B$ are not changed. This will also hold for our data structure.

We shall use these lazy local trees to improve update time to $O\left(\log ^{2} n / \log \log n\right)$. However, it is easy to see that due to the BBSTs in lazy local trees, if we form $\mathcal{C}_{L}$ with these trees, the depth of $\mathcal{C}_{L}$ becomes $O(\log n \log \log n)$. If we use the same approach as in the previous section, we thus increase query and update time by a factor of $\log \log n$. Adding shortcuts to $\mathcal{C}_{L}$ will avoid this slowdown but this gives a data structure with the same bounds as in the previous section.

To handle this, we introduce a new type of lazy local trees. Let $u$ be a non-leaf node of $\mathcal{C}$. A child $v$ of $u$ in $\mathcal{C}$ is said to be heavy if $n(v) \geq n(u) / \log ^{\epsilon} n$ and otherwise it is light; here $\epsilon>0$ is a constant that we may pick as small as we like.

Our lazy local tree $L(u)$ of $u$ is illustrated in Figure 1. It is rooted at $u$ and has two children. One child is the root of a tree $T_{h}(u)$ having the heavy children of $u$ as leaves and the other child is the root of a tree $T_{l}(u)$ having the light children of $u$ as leaves (to simplify 
the description, we assume that $u$ has both light and heavy children; if there were no light (resp. heavy) children, we would simply identify $L(u)$ with $\left.T_{h}(u)\left(\operatorname{resp} . T_{l}(u)\right)\right)$. We call $T_{h}(u)$ the heavy tree (of $u$ ) and it is defined as the local tree from the previous section over the heavy children of $u$; note that its size is asymptotically bounded by the number of heavy children of $u$ which is at most $\log ^{\epsilon} n$. The tree $T_{l}(u)$ is called the light tree (of $u$ ) and it is Thorup's lazy local tree for the set of light children of $u$.

The following lemma shows that when $\mathcal{C}_{L}$ is formed from $\mathcal{C}$ by inserting these lazy local trees, the height of trees in $\mathcal{C}_{L}$ is only a constant factor larger than that in Section 3 .

Lemma 1. The height of $\mathcal{C}_{L}$ (with lazy local trees) is $O(\log n)$.

Proof. Let $u$ be a non-leaf node in $\mathcal{C}$ and let $v$ be one of its children, also in $\mathcal{C}$. If $v$ is a heavy child of $u$ then $v \in T_{h}(u)$ so its depth in $L(u)$ is at most $\log (n(u) / n(v))+1$. Now assume that $v$ is a light child of $u$. Then $v$ belongs to either a bottom tree or the buffer tree of $L(u)$. In the latter case, the depth of $v$ in $L(u)$ is $O(\log \log n)$. In the former case, let $B$ be the bottom tree containing $v$ and let $w$ be a leaf of $B$ maximizing $n(w)$. By definition, the root of $B$ has $\operatorname{rank} \operatorname{rank}(w)$. Hence the depth of $v$ in $L(u)$ is at most $\log (n(u) / n(w))+$ $O(\log \log n) \leq \log (n(u) / n(v))+O(\log \log n)$. This is $O(\log (n(u) / n(v)))$ as $n(v)<n(u) / \log ^{\epsilon} n$ implies $\log \log n=O(\log (n(u) / n(v)))$.

It follows that in both cases, $v$ has depth $O(1+\log (n(u) / n(v)))$ in $L(u)$. The height of $\mathcal{C}$ is at most $\log n$ so by a telescoping sums argument, $\mathcal{C}_{L}$ has height $O(\log n)$, as desired.

\subsection{Maintaining lazy local trees}

Now, let us describe how to maintain lazy local trees of $\mathcal{C}_{L}$ corresponding to changes in $\mathcal{C}$. For technical reasons, we assign ranks to rank path nodes in heavy trees by $\operatorname{rank}\left(v_{i}\right)=\operatorname{rank}\left(r_{i}\right)$ for $i=1, \ldots, k-1$, where $v_{i}$ and $r_{i}$ are defined as in Section 3.4. In the following, rank nodes are nodes that are assigned a rank. Note that every node of $\mathcal{C}_{L}$ is a rank node except non-leaf nodes of a buffer or top tree and nodes of a bottom tree $B$ that are neither leaves nor the root of $B$.

\subsubsection{Merging}

We need to maintain lazy local trees when merging $\mathcal{C}$-nodes and when adding and removing children from $\mathcal{C}$-nodes. We start with merging. Consider two $\mathcal{C}$-nodes $u$ and $v$ that are to be merged into $u$. Denote by $u^{\prime}$ the updated $u$ after the merge. Note that every heavy child of $u^{\prime}$ must be a heavy child of either $u$ or $v$. Hence, we can form $T_{h}\left(u^{\prime}\right)$ by traversing every leaf $w$ of $T_{h}(u)$ and $T_{h}(v)$ and adding it as a leaf of $T_{h}\left(u^{\prime}\right)$ iff $n(w) \geq n\left(u^{\prime}\right) / \log ^{\epsilon} n$. Total time for this is $O\left(\left|T_{h}(u)\right|+\left|T_{h}(v)\right|\right)=O\left(\log ^{\epsilon} n\right)$.

Forming $T_{l}\left(u^{\prime}\right)$ is done as in [10]. The two buffer trees are merged in time bounded by the smaller of the two trees. If the number of leaves of the merged buffer tree exceeds $\log ^{\alpha} n$, it is turned into a bottom tree for $u^{\prime}$, leaving an empty buffer tree. The root of the new bottom tree is paired up with other rank nodes, if needed. We can pay for all buffer tree merges by giving a node $(\log \log n)^{2}$ credits when it switches from not belonging to belonging to a buffer tree: every time it is moved to another buffer tree, we spend $O(\log \log n)$ time for the node and the size of the buffer tree containing the node grows by a factor of at least 2 ; hence the node is moved at most $O(\log \log n)$ times before either being deleted or being moved to a bottom tree. 
We propose a different approach for merging top trees than that of Thorup; we feel ours is simpler as it avoids keeping a special bitmap associated with each top tree node. Assume w.l.o.g. that the top tree $T(u)$ in $T_{l}(u)$ is no bigger than the top tree $T(v)$ in $T_{l}(v)$. For each leaf of $T(u)$ we binary search for a leaf with the same rank in $T(v)$ in $O(\log \log n)$ time. While there are roots with equal ranks, we pair them up as before, finally obtaining the top tree for $T_{l}(w)$. The time for this is $O(|T(u)| \log \log n)$ plus time bounded by the number of new rank nodes created. Below we will show how the creation of rank nodes are paid for when they are deleted. With an amortized analysis similar to that above for buffer trees, we can pay for all top tree updates if we assign $(\log \log n)^{2}$ credits to a node when it switches from not appearing to appearing as a leaf in a top tree, and if we borrow $(\log \log n)^{2}$ credits whenever we delete a leaf of a top tree (thereby borrowing from a new rank node) and distribute these borrowed credits evenly among the remaining leaves.

Since $n\left(u^{\prime}\right)=n(u)+n(v)$, we may have some leaves $w \in T_{h}(u) \cup T_{h}(v)$ with $n(w)<$ $n\left(u^{\prime}\right) / \log ^{\epsilon} n$ and hence $w$ should belong to $T_{l}\left(u^{\prime}\right)$. All such nodes are added to the buffer tree; as before, this tree is turned into a bottom tree if it gets more than $\log ^{\alpha} n$ leaves. Total time for this is $O\left(\log ^{\epsilon} n \log \log n\right)$ (plus time bounded by the number of new rank nodes created) since at most $2 \log ^{\epsilon} n$ nodes need to be moved from $T_{h}(u) \cup T_{h}(v)$.

Finally, let $p$ be the parent of $u$ and $v$ in $\mathcal{C}$; below we will add shortcuts that allow us to identify $p$ from $u$ (equivalently from $v$ ) in $O(\log n / \log \log n$ ) time (Lemma 3). We remove $u$ and $v$ as leaves of $L(p)$ and then we add $u^{\prime}$ as a leaf of $T_{h}(p)$ if $n\left(u^{\prime}\right) \geq n(p) / \log ^{\epsilon} n$ and otherwise we add $u^{\prime}$ as leaf of the buffer tree in $T_{l}(p)$. This takes $O(\log \log n)$ time since both the buffer tree and $T_{h}(p)$ have poly-logarithmic size and their roots have depth $O(\log \log n)$ in $L(p)$.

\subsection{Removing a child}

Consider removing a $\mathcal{C}$-node child $v$ of a $\mathcal{C}$-node $u$ and adding it as a child of a new $\mathcal{C}$-node $w$ which is added as a child of the parent $\mathcal{C}$-node $p$ of $u$. We first focus on removing $v$ and we let $u^{\prime}$ denote $u$ after this update.

Assume first that $v \in T_{h}(u)$. After removing $v$ from $T_{h}(u)$, we have $n\left(u^{\prime}\right)=n(u)-n(v)$ and hence some nodes may need to be moved from $T_{l}(u)$ to $T_{h}(u)$ in order to form $L\left(u^{\prime}\right)$. Identifying such nodes in the buffer tree of $T_{l}(u)$ can be done in $O(\log \log n)$ time per node. Now suppose $w$ is a leaf of a bottom tree $B$ that needs to be moved to $T_{h}(u)$. Let $b$ be the root of $B$ and let $a$ be the leaf of the top tree of $T_{l}(u)$ having $b$ as descendant. Since $\operatorname{rank}(b) \geq \operatorname{rank}(w) \geq\left\lfloor\log \left(n\left(u^{\prime}\right) / \log ^{\epsilon} n\right)\right\rfloor>\lfloor\operatorname{rank}(a)-\epsilon \log \log n\rfloor$ and since ranks are strictly decreasing on the path from $a$ to $b, b$ has depth at most $\lceil\epsilon \log \log n\rceil$ in the subtree of $T_{l}(u)$ rooted at $a$. A DFS from $a$ identifies all bottom tree roots with at most this depth in $O\left(\log ^{\epsilon} n\right)$ time; let $L_{a}$ be the set of leaves in these bottom trees that need to be moved to $T_{h}(u)$. Using binary search in the $O\left(\log ^{\epsilon} n\right)$ bottom trees, we identify $L_{a}$ and move it to $T_{h}(u)$ in time $O\left(\left(\log ^{\epsilon} n+\left|L_{a}\right|\right) \log \log n\right)$. Since all rank leaves of the top tree of $T_{l}(u)$ have distinct ranks, there are only $O(\log \log n)$ choices for $a$. Also, the maximum number of leaves to be moved is bounded by the number of leaves of $T_{h}\left(u^{\prime}\right)$ which is at $\operatorname{most} \log ^{\epsilon} n$. Total time is thus $O\left(\log ^{\epsilon} n(\log \log n)^{2}\right)$.

We may also need to move $u$ in $L(p)$. If $u$ belongs to a bottom tree in $T_{l}(p)$, we move it to the buffer tree as we do not allow ranks of leaves in bottom trees to change. We also move $u$ to the buffer tree if $u \in T_{h}(p)$ and $n\left(u^{\prime}\right)<n(p) / \log ^{\epsilon} n$. As we saw for merge, the time for this is $O(\log n / \log \log n)$. 
As in [10, we need to do more global updates whenever removing a leaf from a bottom tree $B$ of $T_{l}(u)$ reduces the maximum rank of leaves in $B$ and hence the rank of the root $b$ of $B$. We use a similar approach and amortized analysis as Thorup here: first, delete all rank nodes from $b$ to the ancestor leaf $a$ of the top tree of $T_{l}(u)$. Then pair nodes of equal rank as before. For the amortized analysis, we can assume that the graph $G$ ends with no edges so all rank nodes end up being deleted and we can amortize creation of rank nodes in $T_{l}(u)$ over deletion of rank nodes in $T_{l}(u)$. A rank node is only deleted when a bottom tree root has its rank reduced. Since a rank is at most $\log n$, a particular bottom tree can have its root rank reduced at most $\log n$ times (nodes are never added to a bottom tree and ranks of bottom tree leaves do not change) so in total it gives rise to at most $\log ^{2} n$ rank node deletions for that bottom tree. But since a bottom tree starts out with $(\log n)^{\alpha}$ leaves that will all be deleted eventually, we can amortize each rank node deletion over $(\log n)^{\alpha-2}$ deletions of bottom tree leaves. When removing child $v$, we delete at $\operatorname{most} \log ^{\epsilon} n$ leaves from bottom trees of $T_{l}(u)$ in order to form $L\left(u^{\prime}\right)$ so we can amortize each rank node deletion over $(\log n)^{\alpha-2-\epsilon}$ deletions of children in $\mathcal{C}$. Hence if we pick constant $\alpha \geq 2+\epsilon$, we can afford to pay for rank node deletions and also to pay for the $(\log \log n)^{2}$ credits that may have been borrowed from a rank node during a merge.

It remains to consider the case $v \in T_{l}(u)$. Above we showed how to efficiently remove up to order $\log ^{\epsilon} n$ leaves from $T_{l}(u)$ so clearly the single leaf $v$ can also be removed efficiently. We then move additional leaves from $T_{l}(u)$ to $T_{h}(u)$ and move $u$ to $T_{h}(p)$ or to the buffer tree of $T_{l}(p)$, as above.

\subsection{Adding a child}

Now consider adding $v$ as a child of $w$ and $w$ as a child of $p$. The former is trivial as $w$ has no children before adding $v$. If $n(v) \geq n(p) / \log ^{\epsilon} n$, we add $w$ to $T_{h}(p)$ and otherwise we add it to the buffer tree of $T_{l}(p)$. Given $p$, total time for this is $O(\log \log n)$.

\subsection{Shortcutting}

In order to get our $\log \log n$ speed-up for updates and queries, we need to be able to traverse $\mathcal{C}_{L}$ faster. Thorup [10] introduced a system of shortcuts for quickly identifying certain edges incident to clusters. This will not suffice in our approach since for our search procedures, we also need to move quickly from a leaf of $\mathcal{C}_{L}$ to its ancestor level $i$ node in order to identify the associated level $i$ cluster, for some $i$. We therefore introduce a different system of shortcuts in the following. To avoid skipping past a level $i$ node with these shortcuts, our data structure associates, for each node of a heavy tree $T_{h}(u)$, the level $\ell(u)$ of $u$. We can easily extend the data structure for lazy local trees to maintain these values within the same time bound since $T_{h}(u)$ has only size $O\left(\log ^{\epsilon} n\right)$.

Let us color each node of $\mathcal{C}_{L}$ either white or black. For the coloring below, we define a black-induced child of a node $u \in \mathcal{C}_{L}$ to be a black descendant $v$ of $u$ such that all interior nodes on the path from $u$ to $v$ in $\mathcal{C}_{L}$ are white. If $u$ is black, we add a shortcut between $u$ and each of its black-induced children. The shortcut is directed to $u$, allowing us to move quickly up in $\mathcal{C}_{L}$. The black-induced parent of a node is defined similarly. Note that the shortcuts (with directions reversed) form a forest of rooted trees over the black nodes.

Now, let us define the coloring of nodes of $\mathcal{C}_{L}$. The following nodes are colored black:

1. every $\mathcal{C}$-node $u$ with $\ell(u)=i\lfloor\epsilon \log \log n\rfloor$ for some integer $i$, 
2. every rank node $u$ having a parent rank node $v$ in $\mathcal{C}_{L} \operatorname{such}$ that $\operatorname{rank}(u) \leq i\lfloor\epsilon \log \log n\rfloor<$ $\operatorname{rank}(v)$ for some integer $i$,

3. every leaf of $\mathcal{C}_{L}$ and of every buffer, bottom, and top tree, and

4. every node of a buffer, bottom, and top tree whose depth in that tree is divisible by $\lfloor\epsilon \log \log n\rfloor$ (in particular, every root of such a tree is black).

A black node is of type 1, 2, 3, and/or 4, depending on these four cases. All other nodes are colored white. For performance reasons, we shall only maintain edge-bitmaps for black nodes. Lemma 3 below shows that these shortcuts give a $\log \log n$ speed-up when moving up a tree of $\mathcal{C}_{L}$. We first need the following result.

Lemma 2. Ranks are non-decreasing along any simple leaf-to-root path in $\mathcal{C}_{L}$. Between any two consecutive $\mathcal{C}$-nodes on such a path, there are at most two pairs of nodes of equal rank.

Proof. The first part of the lemma will follow if we can show that ranks are non-decreasing along any simple leaf-to- $u$ path $P$ in a lazy local tree $L(u)$. This is clearly the case for leaves in $T_{h}(u)$. A leaf $v$ in $T_{l}(u)$ either belongs to a bottom or buffer tree $T$. Assume the former since otherwise, $u$ and $v$ are the only rank nodes on the $v$-to- $u$ path $P$ and since both are $\mathcal{C}$-nodes, $\operatorname{rank}(u) \geq \operatorname{rank}(v)$.

Only the first node $v$ and last node $r$ of subpath $P[v, r]=T \cap P$ are rank nodes. Since $r$ is the root of $T$, it has maximum rank among leaves in $T$ so $\operatorname{rank}(v) \leq \operatorname{rank}(r)$. Let $l$ be the leaf of the top tree of $L(u)$ belonging to $P$. All nodes on $P[r, l]$ belong to a rank tree so ranks are increasing along this subpath. For the subpath $P[l, u]$, only $l$ and $u$ are rank nodes. Let $L$ be the set of leaves of $T_{l}(u)$ formed by picking one of maximum rank from each bottom tree descending from $l$. Then $\operatorname{rank}(l) \leq\left\lfloor\log \left(\sum_{u^{\prime} \in L} n\left(u^{\prime}\right)\right)\right\rfloor \leq\lfloor\log n(u)\rfloor=\operatorname{rank}(u)$. This $\operatorname{shows}$ the first part of the lemma.

For the second part, let $u$ and $v$ be $\mathcal{C}$-nodes where $v$ is a child of $u$. Assume first that $v$ is a leaf of $T_{h}(u)$. Ranks are strictly increasing on the path from $v$ to the root $r$ of the rank tree containing $v$. Ranks are also strictly increasing along the rank path in $T_{h}(u)$. Hence, there are at most two pairs of equal rank nodes in $\mathcal{C}_{L}$ between $u$ and $v$, namely $r$ and its parent and the root of $T_{h}(u)$ and $u$. Now consider the case where $v$ is a leaf of $T_{l}(u)$ and again assume it belongs to a bottom tree $B$. Let $r$ be the root of $B$ and let $l$ be the leaf of the top tree which is an ancestor of $v$. Then again, since ranks are strictly increasing along any leaf-to-root path in a rank tree, there can be at most two equal-rank pairs between $u$ and $v$, namely $(v, r)$ and $(l, u)$. This completes the proof.

Lemma 3. Given $\mathcal{C}_{L}$ with shortcuts, given a level $i$, and given a $\mathcal{C}$-node of $\mathcal{C}_{L}$ with an ancestor level $i$ node, we can identify this ancestor in $O(\log n / \log \log n)$ time.

Proof. Let $v$ be the given node. To identify the ancestor level $i$ node $u$ of $v$, we start by traversing the $v$-to-root path of the tree in $\mathcal{C}_{L}$ containing $v$ and we stop if we reach $u$ or a black node. Since $v$ is a $\mathcal{C}$-node and since light trees have black leaves, all nodes visited are rank nodes. Lemma 2 then implies that we visit at most $O(\log \log n)$ nodes before stopping. Hence, the traversal takes $O(\log \log n)$ time. Assume that we reach a black node $b_{1}$ as we are done if we reach $u$.

From $b_{1}$ we traverse shortcuts until we get to the lowest-depth black node $b_{2}$ having $u$ as ancestor. Finally we traverse the $b_{2}$-to-root path in $\mathcal{C}_{L}$ until we reach $u$. The latter takes $O(\log \log n)$ time by an argument similar to the above. 
Next we show that there are $O(\log n / \log \log n)$ shortcuts between $b_{1}$ and $b_{2}$. Since $\mathcal{C}$ has $\operatorname{logarithmic}$ height, there are only $O(\log n / \log \log n)$ shortcut endpoints of type 1 . Lemma 2 implies the same bound for shortcut endpoints of type 2. If a shortcut ends at a type 3 node $b$ which is a leaf of a buffer or bottom tree, it means that we enter a light tree $T_{l}(w)$. We encounter only one additional type 3 node in $T_{l}(w)$, namely a leaf of a top tree. Since $n(w)>n(b) \log ^{\epsilon} n$ we have $\operatorname{rank}(w)>\operatorname{rank}(b)+\lfloor\epsilon \log \log n\rfloor$ and since a rank is at most $\log n$, Lemma 2 implies that we encounter no more than $O(\log n / \log \log n)$ type 3 nodes between $b_{1}$ and $b_{2}$. Finally, this bound on the number of type 3 nodes and Lemma 1 give the same asymptotic bound on the number of type 4 nodes.

What remains is to describe how to avoid jumping past $u$ when traversing the shortcuts. Let $\left(b_{2}, b_{3}\right)$ be the shortcut that jumps past $u$, if any. Since leaves of light trees are black, $b_{3}$ must belong to some heavy tree $T_{h}\left(w_{b_{3}}\right)$. If $b_{2}$ belongs to a light tree, it must belong to the root of the top tree in $T_{l}(u)$ since that root is black. We can avoid this case as follows: whenever we reach the root of a top tree, its parent is a $\mathcal{C}$-node and we compare its level with $i$ to determine whether we should continue with the shortcuts.

Now, consider the case where $b_{2}$ belongs to a heavy tree $T_{h}\left(w_{b_{2}}\right)$. Recalling that for every node of a heavy tree $T_{h}(w)$ we keep the level $\ell(w)$ of $w$, we can check that $\ell\left(w_{b_{2}}\right) \leq i<\ell\left(w_{b_{3}}\right)$ to detect that $a$ is the last node that we should visit with shortcuts. This completes the proof.

\subsection{Induced shortcuts}

Lemma 3 allows us to speed up part of our search procedure, namely identifying the endpoints (level $(i+1)$ clusters) of an edge in a multigraph $M_{i}$ from the endpoints of the corresponding edge in $G$; we can do this in $O(\log n / \log \log n)$ time per endpoint. We also need a faster iterator for level $i$-edges incident to explored level $(i+1)$ clusters. We focus on this in the following.

Define an $i$-induced forest $\mathcal{F}_{i}$ as in [10]: its $i$-induced leaves are the leaves of $\mathcal{C}_{L}$ with an incident level $i$-edge. Its $i$-induced roots are the level $(i+1)$ nodes of $\mathcal{C}_{L}$ having descendant $i$-induced leaves. Its $i$-induced branch nodes are the nodes of $\mathcal{C}_{L}$ with both children have descending $i$-induced leaves. The $i$-induced parent of an $i$-induced node is its nearest $i$ induced ancestor. This defines $\mathcal{F}_{i}$.

A straightforward level $i$-iterator performs a DFS in a tree of $\mathcal{F}_{i}$. However, maintaining the edges of $\mathcal{F}_{i}$ will be too expensive. Instead, we introduce a new system of shortcuts in $\mathcal{C}_{L}$ that will allow the DFS to move between any two incident $i$-induced nodes of $\mathcal{F}_{i}$ in $O(\log n / \log \log n)$ time. Since a tree of $\mathcal{F}_{i}$ is binary, the number of branch nodes of $T$ is bounded by the number of leaves of $T$, so this will give a level $i$-iterator with $O(\log n / \log \log n)$ amortized time per level $i$-edge. In the following, we define the new shortcuts. Refer to the following types of nodes of $\mathcal{C}_{L}$ as special:

1. every $\mathcal{C}$-node $u$ with $\ell(u)=i\lfloor\log \log n\rfloor\lfloor\epsilon \log \log n\rfloor$ for some integer $i$,

2. every leaf of $\mathcal{C}_{L}$, and

3. every rank node $u$ of a light tree with $\operatorname{rank}(u)=i\lfloor\log \log n\rfloor\lfloor\epsilon \log \log n\rfloor$ for some integer $i$.

Note that every special node is black. Also note that we defined type 3 special nodes using equality rather than inequality as for type 2 black nodes. This suffices since ranks increase 
by 1 as we move up rank nodes of a light tree; this is not the case in heavy trees where ranks can increase by larger values along a rank path. For a special node $u$, define a special child of $u$ to be a descendant special node $v$ such that all nodes between $u$ and $v$ are not special. Special parents are defined similarly. For any level $i$, if there is a unique special child $v$ of $u$ for which edge $(v)[i]=1$, we add a shortcut (bidirected pointer) between $u$ and $v$. To distinguish these shortcuts from those of Section 4.5, we refer to the former as $i$-induced shortcuts or just induced shortcuts and the latter as standard $i$-shortcuts or just standard shortcuts. Observe that for all $i$-induced shortcuts $(a, b)$, where $b$ is a special child of $a$, there is an edge in $\mathcal{F}_{i}$ from $a$ or an ancestor of $a$ to $b$ or a descendant of $b$. For each special node $u$, we keep a BBST with a leaf for each $i$ containing the $i$-induced shortcuts to a special child and/or parent (if they exist).

\subsection{Faster iterator}

Now let us present the level $i$-iterator. It starts at the root $v$ of a tree in $\mathcal{F}_{i}$, i.e., $v$ is a level $(i+1)$ node of $\mathcal{C}_{L}$. It performs a DFS of the subtree of $\mathcal{C}_{L}$ rooted at $v$ with the following modification: if it visits a black node $w$ for which edge $(w)[i]=0$, it backtracks; if it visits a special node $w^{\prime}$ with an $i$-induced shortcut to a special child, it visits this special child instead of the children of $w^{\prime}$ in $\mathcal{C}_{L}$. When it reaches a leaf $l$ of $\mathcal{C}_{L}$, it identifies the group of incident level $i$-edges with a binary search in the BBST associated with $l$ and then iterates over these edges. This completes the description of the level $i$-iterator. Lemma 6 below shows the performance of the level $i$-iterator. To prove it, we need two additional lemmas.

Lemma 4. Any node of $\mathcal{C}_{L}$ has only $O\left((\log n)^{3 \epsilon}\right)$ black-induced children.

Proof. Let $u$ be a node of $\mathcal{C}_{L}$. If $u$ is a non-leaf node of a bottom, buffer, or top tree, the lemma follows from the definition of type 3 and 4 black nodes. Otherwise, $u$ is a rank node. For any black-induced child $v$ of $u$, Lemma 2 and the definition of type 1 and 2 black nodes imply that $v$ has depth at most $3 \epsilon \log \log n$ in the subtree of $\mathcal{C}_{L}$ rooted at $u$. As $\mathcal{C}_{L}$ is binary, the lemma follows.

Lemma 5. For any $i$-induced shortcut $(a, b)$, the simple $a$-to-b path in $\mathcal{C}_{L}$ has length $O\left((\log \log n)^{4}\right)$.

Proof. Let $P$ be the simple $a$-to- $b$ path in $\mathcal{C}_{L}$. Clearly, $P$ contains only $O\left((\log \log n)^{2}\right) \mathcal{C}$ nodes. Let $P^{\prime}$ be a subpath of $P$ containing no $\mathcal{C}$-nodes. Then $P^{\prime}$ is either contained in a heavy or a light tree. In the former case, $\left|P^{\prime}\right|=O(\log \log n)$. In the latter case, we encounter at most $O(\log \log n)$ nodes of buffer, bottom, and top trees on $P^{\prime}$. Since consecutive rank nodes of $P^{\prime}$ differ in rank by exactly 1 (as they all belong to a light tree and hence to a rank tree), we encounter at most $O\left((\log \log n)^{2}\right)$ rank nodes on $P^{\prime}$ so $\left|P^{\prime}\right|=O\left((\log \log n)^{2}\right)$.

Lemma 6. The level $i$-iterator above traverses a tree in $\mathcal{F}_{i}$ with $k$ leaves in $O(k \log n / \log \log n)$ time. The time to visit the first $k^{\prime}$ leaves is $O\left(k^{\prime} \log n / \log \log n+\log n\right)$.

Proof. Correctness follows easily from the definition of edge-bitmaps, $i$-induced shortcuts, and BBSTs associated with leaves of $\mathcal{C}_{L}$ so let us focus on the time bound to traverse a $k$-leaf tree $T$ in $\mathcal{F}_{i}$. Let $T_{L}$ be the tree in $\mathcal{C}_{L}$ obtained by replacing each edge $(a, b) \in T$ with the corresponding simple path $P$ in $\mathcal{C}_{L}$ between $a$ and $b$. By Lemmas 1 and 2, there can only be $O\left(\log n /(\log \log n)^{2}\right)$ special nodes on such a path $P$. Hence, since $T$ has no degree 2 -vertices, the total number of special nodes and hence $i$-induced shortcuts traversed by the 
level $i$-iterator in $T_{L}$ is $O\left(k \log n /(\log \log n)^{2}\right)$. For each special node visited, $O(\log \log n)$ time is spent on binary search to find the next $i$-induced shortcut, if it exists. Hence, the total time spent on visiting special nodes and traversing $i$-induced shortcuts is $O(k \log n / \log \log n)$.

We will now show that the number of additional nodes visited by the DFS is $O\left(k(\log n)^{3 \epsilon}(\log \log n)^{4}\right)$. Since only constant time is spent for each such node, this will show the first part of the lemma. First we bound the number of visited nodes of $T_{L}$ which are not special. Let $(a, b)$ and $P$ be as above. If we traverse $P$ from $a$ then it follows from Lemma 5 that after at most $O\left((\log \log n)^{4}\right)$ nodes, we will reach either $b$ or a special node $a^{\prime}$. Similarly, if we traverse $P$ from $b$ then after at most $O\left((\log \log n)^{4}\right)$ nodes, we will reach either $a$ or a special node $b^{\prime}$. If $a^{\prime}$ and $b^{\prime}$ exist then all nodes visited by the DFS on $P\left[a^{\prime}, b^{\prime}\right]$ are special nodes connected by $i$-induced shortcuts. Summing over all such paths $P$, it follows that the total number of nodes visited on $T_{L}$ which are not special is $O\left(k(\log \log n)^{4}\right)$.

Finally, let us bound the number of nodes of $\mathcal{C}_{L}$ visited by the DFS which are not on $T_{L}$. Consider a visited node $u \in T_{L}$ and let $v \notin T_{L}$ be a visited node such that $u$ is the nearest ancestor of $v$ belonging to $T_{L}$. Note that there is no $i$-induced shortcut from $u$ to a special child since then the DFS would have traversed this shortcut instead of the children of $u$ in $\mathcal{C}_{L}$. In particular, there are only $O\left(k(\log \log n)^{4}\right)$ choices for $u$. Furthermore, all interior nodes on the simple path from $u$ to $v$ in $\mathcal{C}_{L}$ are white since any black node $w$ would have edge $(w)[i]=0$ (as $w \notin T_{L}$ ), meaning that the DFS would have backtracked before reaching $v$. By Lemma 4 , there are only $O\left((\log n)^{3 \epsilon}\right)$ choices for $v$ for each $u$. Hence, the total number of nodes visited which are not on $T_{L}$ is $O\left(k(\log n)^{3 \epsilon}(\log \log n)^{4}\right)$. This shows the first part of the lemma.

For the second part, consider a partially grown DFS tree $T^{\prime}$ which has visited $k^{\prime}$ leaves. For every node of $T^{\prime}$ having two children, at least one of the two subtrees rooted at the children is fully explored. Hence, $T^{\prime}$ consists of a path $P$ from the root of $T$ to a leaf of $T$ with fully explored subtrees attached to $P$. The same argument as above shows that the total time to explore these subtrees is $O\left(k^{\prime} \log n / \log \log n\right)$. By Lemma 1, it takes $O(\log n)$ time to explore $P$ (the number of special nodes on $P$ is $O\left(\log n /(\log \log n)^{2}\right)$ so we only spend a total of $O(\log n / \log \log n)$ time on binary searches for these nodes).

It follows from Lemma 6 that the level $i$-iterator spends $O(\log n / \log \log n)$ amortized time per edge visited plus additional $O(\log n)$ time if a replacement path was found (if such a path is not found, an entire tree in $\mathcal{F}_{i}$ is traversed). The $O(\log n / \log \log n)$ amortized time per edge is paid for by the increase in the level of the edge and the $O(\log n)$ time is paid for by the deletion of an edge in $G$ since at most one replacement path is found for such an edge.

It remains to describe how colors, shortcuts (standard and induced) and edge-bitmaps are maintained when $\mathcal{C}$ (and hence $\mathcal{C}_{L}$ ) is updated and when edges of $G$ are added/removed or change level. First we deal with changes to $\mathcal{C}$. The following lemma will prove useful.

Lemma 7. Given edge-bitmaps of black nodes and given a special node $u$, we can find the induced shortcuts between $u$ and its special parent (if any) in $O(\log n)$ time. For any $i$, we can find the $i$-induced shortcut from $u$ to a special child or determine that no such shortcut exists in $O\left((\log n)^{3 \epsilon}(\log \log n)^{4}\right)$ time.

Proof. We first walk up $\mathcal{C}_{L}$ from $u$ to identify its special parent $p$. By Lemma 5 , this takes $O\left((\log \log n)^{4}\right)$ time. Then we perform a DFS in the subtree of $\mathcal{C}_{L}$ rooted at $p$ and backtrack if we encounter $u$ or a black node which is not an ancestor of $u$. If any such black node is encountered for which the $i$ th bit of its edge-bitmap is 1 then we know that there should not be an $i$-induced shortcut between $u$ and $p$. Otherwise there should be iff edge $(u)[i]=1$. 
Let $m$ be the bitmap obtained by taking the bitwise 'or' of the edge-bitmaps of visited black nodes not on the $u$-to- $p$ path. By Lemmas 4 and 5 , finding $m$ takes $O\left((\log n)^{3 \epsilon}(\log \log n)^{4}\right)$ time. Now, there is an $i$-induced shortcut between $u$ and $p$ iff $m[i]=0$ and edge $(u)[i]=1$. Hence, all induced shortcuts between $u$ and $p$ can be found in $O(\log n)$ time.

To find the $i$-induced shortcut (if any) to a special child of $u$, we make a DFS from $u$ which backtracks when reaching a black node. Suppose exactly one visited black node $w$ has edge $(w)[i]=1$ (otherwise, there is no $i$-induced shortcut). If $w$ is special, we have identified the $i$-induced shortcut $(w, u)$. Otherwise, we recurse on $w$. As above, total time for this is $O\left((\log n)^{3 \epsilon}(\log \log n)^{4}\right)$.

\subsection{Structural changes}

Let us now describe how shortcuts and edge-bitmaps are maintained after structural changes to $\mathcal{C}_{L}$. It follows from Lemma 4 that for each update to $\mathcal{C}_{L}$, we can update colors, standard shortcuts and edge-bitmaps in $O\left((\log n)^{3 \epsilon}\right)$ time. From the results in Section 4.2, this will not affect the overall time bound (if we pick constant $\alpha$ sufficiently large). In the following, we thus only consider updating induced shortcuts.

We shall restrict our attention to structural changes caused by a delete-operation as insert corresponds to merging two clusters (or none), a type of update that needs to be supported during a delete.

Recall that after a delete-operation, $\mathcal{C}$ is updated as follows: some children of a node $u$ are removed and merged into a single node; this node is either added as a child of $u$ (if a replacement path was found) or it is added as a child of a new node $u^{\prime}$ which is added as a child of the parent $p$ of $u$ and the process is repeated recursively on $p$ (if a replacement path was not found). We observe that all $\mathcal{C}$-nodes whose children are updated are contained in two leaf-to-root paths in $\mathcal{C}$ after the delete-operation has been executed.

There are two types of induced shortcuts that need to be updated, those incident to a type 1 special node and those descending from a type 3 special node and not ascending from a type 1 special node (see definitions of types in Section 4.6). Below we show how to update the latter.

For the former, it follows from the above that we only need to focus on type 1 special nodes on a leaf-to-root path $P$ in $\mathcal{C}_{L}$ (there are two paths but they are handled in the same manner). Let $u_{1}, \ldots, u_{k}$ be the sequence of special nodes as we move up $P$ during the delete-operation (some of them may be new or merged nodes and hence do not exist before the delete-operation). By Lemma 7 , we can find all induced shortcuts descending from $u_{1}$ in $O\left((\log n)^{1+3 \epsilon}(\log \log n)^{4}\right)$ time. When we reach $u_{j}, j>1$, we compute induced shortcuts between $u_{j-1}$ and $u_{j}$. By Lemma 7 this takes $O(\log n \log \log n)$ time (including binary searches in the BBSTs of $u_{j-1}$ and $\left.u_{j}\right)$ for a total of $O\left(\log ^{2} n / \log \log n\right)$ over all $j$ which the delete-operation can pay for. We also compute induced shortcuts descending from $u_{j}$ for those $i$ for which edge $\left(u_{j}\right)[i]=1$ and edge $\left(u_{j-1}\right)[i]=0$. Total time over all $j$ is $O\left((\log n)^{1+3 \epsilon}(\log \log n)^{4}\right)$ since if edge $\left(u_{j}\right)[i]=1$ then edge $\left(u_{j^{\prime}}\right)[i]=1$ for all $j^{\prime}>j$, implying that the second part of Lemma 7 is applied at most once for each $i$. Note that all $i$-induced shortcuts from $u_{j}$ to a special child which have not been identified by the second part of Lemma 7 must have edge $\left(u_{j}\right)[i]=\operatorname{edge}\left(u_{j-1}\right)[i]=1$ and hence must connect $u_{j}$ to $u_{j-1}$ which we have computed above. Hence, we correctly compute all induced shortcuts incident to special nodes on $P$ and we do so within the desired time bound.

Now we show how to maintain induced shortcuts descending from a type 3 special node 
$p$ and not ascending from a type 1 special node. We may assume that $p$ is not on one of the leaf-to-root paths considered above. Then the only structural changes to $\mathcal{C}_{L}$ that may require such shortcuts to be updated are

1. a type 3 special node is created or deleted, or

2. a leaf is removed from a bottom tree in the light tree containing $p$.

A type 3 special node can pay any polylogarithmic amount when it is created/deleted so consider updating induced shortcuts descending from $p$ when a leaf $u$ is removed from a bottom tree $B$ in the light tree $T_{l}(w)$ containing $p$. To handle this case, we will assume that each rank node of $T_{l}(w)$ has $\log n / \log \log n$ credits for each $i$ for which it is an $i$-induced branch node. To see that this assumption can be made, first observe that when a buffer tree is turned into a bottom tree, it can pay any polylogarithmic amount if we pick $\alpha$ sufficiently big. This is also the case when a new rank node of $T_{l}(w)$ is created/deleted. Since we never add but only remove leaves from bottom trees, the only other way a rank node of $T_{l}(w)$ can become an $i$-induced branch node is if some edge of $G$ has its level increased to $i$. Such an edge can only contribute with two $i$-induced branch nodes to $\mathcal{F}_{i}$ (one for each of its endpoints) so we may add $\log n / \log \log n$ credits to the two new $i$-induced branch nodes which the level increase can pay for. This shows the desired.

Let $\operatorname{branch}(u)$ be the bitmap where $\operatorname{branch}(u)[i]=1$ iff there is an $i$-induced branch node $v \neq u$ on the path from $u$ to $p$. By Lemmas 4 and 5, we can form $\operatorname{branch}(u)$ in $O\left((\log n)^{3 \epsilon}(\log \log n)^{4}\right)$ time which the removal of $u$ from $B$ can pay for. For each $i$ for which $\operatorname{branch}(u)[i]=1$, the removal of $u$ may require us to compute an $i$-induced shortcut descending from $p$. Since a branch node is removed in the process, we can spend its credits to pay for applying Lemma 7 to find this shortcut. We can binary search for each of the $k$ 1-entries of $\operatorname{branch}(u)$ in $O(k \log \log n)$ time; for instance, to determine whether the lower half of $\operatorname{branch}(u)$ has any 1-bits, we can take the bitwise 'and' of $\operatorname{branch}(u)$ and a precomputed bitmap having 1-bits in the lower half and 0-bits in the upper half. The 'and' is 1 iff there are 1-bits in the lower half of $\operatorname{branch}(u)$.

Now consider an $i$ for which $\operatorname{branch}(u)[i]=0$. We may assume that a $\operatorname{bitmap} \operatorname{induced}(p)$ associated with $p$ is maintained where induced $(p)[j]=1$ iff $p$ has a $j$-induced shortcut to a special child. If edge $(u)[i]=0$ or $\operatorname{induced}(p)[i]=0$, nothing needs to be done for $i$ so assume edge $(u)[i]=1$ and induced $(p)[i]=1$. Then we delete the $i$-induced shortcut descending from $p$. If there is an $i$-induced shortcut from $p$ to its special parent $p^{\prime}$, we remove it too and recurse

on $p^{\prime}$; the recursion stops when we reach a special node $q$ without an $i$-induced shortcut to its special parent $q^{\prime}$. Each induced shortcut can be removed in $O(\log \log n)$ time using binary search in the associated BBSTs. By adding $\log \log n$ credits to an induced shortcut when it is created, we can pay for all deletions of induced shortcuts. If $q^{\prime}$ is a rank node of $T_{l}(w)$, we may need to add an $i$-induced shortcut from $q^{\prime}$ to a special child. This can only happen if an $i$-induced branch node between $q$ and $q^{\prime}$ disappears and as above, we can spend its credits to pay for finding this shortcut.

\section{$4.9 \quad$ Non-structural changes}

Above we dealt with updates of shortcuts due to structural changes in $\mathcal{C}_{L}$. We now handle updates when leaves of $\mathcal{F}_{i}$ appear or disappear due to edge level changes. 
Edge deletions: When a level $i$-edge $e$ is deleted (possibly due to its level being increased to $i+1$ ), edge $(u)[i]$ might change from 1 to 0 for one of its endpoints $u$ which will then no longer be an $i$-induced leaf of $\mathcal{F}_{i}$. We describe how to update other edge-bitmaps accordingly and remove some of the $i$-induced shortcuts. The following is done for $u$. If there are still level $i$-edges incident to $u$ then no updates are needed. Otherwise, all $i$-induced shortcuts on the simple path in $\mathcal{C}_{L}$ from $u$ to its $i$-induced parent $p$ should be removed. Since $u$ is a leaf of $\mathcal{C}_{L}$, it is a special node. We traverse $i$-induced shortcuts from $u$ to ancestors until we reach a special node $v$ without an $i$-induced shortcut to an ancestor. Since $u$ is the only $i$-induced leaf below $v$, we delete all shortcuts traversed. We also set edge $(v)[i] \leftarrow 0$ for all black nodes $v$ between $u$ and $v$ (including $v)$ in $O(\log n / \log \log n)$ time by traversing standard $i$-shortcuts between $u$ and $v$.

We then traverse black nodes up from $v$ in $\mathcal{C}_{L}$ and stop if we find a special node $w$. Whenever we visit a black node $b$, we perform a DFS in the subtree of $\mathcal{C}_{L}$ rooted at $b$, backtracking at descending black nodes. If each black node $b^{\prime}$ visited below $b$ has edge $\left(b^{\prime}\right)[i]=$ $0, u$ was the only leaf of $\mathcal{C}_{L}$ below $b$ with an incident level $i$-edge so we set edge $(b)[i] \leftarrow 0$ and proceed up to the next black node. Conversely, if some black node $b^{\prime}$ visited below $b$ has edge $\left(b^{\prime}\right)[i]=1, p$ must be below $b$ and no more bitmaps need to be updated.

Having updated the bitmaps and removed all $i$-induced shortcuts below $v$, we need to check if an $i$-induced shortcut should be added from $w$ to one of its descendants. By Lemma 7 , this takes $O\left((\log n)^{3 \epsilon}(\log \log n)^{4}\right)$ time which can be paid for by the deletion of $e$; here we can also afford to add $\log \log n$ credits to the shortcut if it was added.

Edge insertions Now suppose a level $i$-edge $e$ is inserted. To update edge-bitmaps and add new $i$-induced shortcuts, we do the following for each endpoint $u$ of $e$. If other level $i$-edges are incident to $u$ then nothing needs to be done as $u$ is already a leaf of $\mathcal{F}_{i}$ so assume otherwise. Inserting $e$ corresponds to updating $\mathcal{F}_{i}$ by adding a new edge $(u, p)$, where $p$ is the $i$-induced parent of $u$. Hence, we need to add $i$-induced shortcuts between $u$ and $p$. Suppose $u$ has an ancestor black node $v$ such that edge $(v)[i]=1$. We traverse standard shortcuts up from $u$ and stop when we identify the first such $v$. Let $u_{1}, \ldots, u_{k}$ be the ordered (possibly empty) sequence of special nodes visited from $u$ to $v$. Since $v$ already has a descending leaf incident to a level $i$-edge and $u_{k}$ does not, $p$ must be on the $u_{k}$-to- $v$ path in $\mathcal{C}_{L}$. Hence, the new $i$-induced shortcuts to be added are $\left(u_{1}, u_{2}\right),\left(u_{2}, u_{3}\right), \ldots,\left(u_{k-1}, u_{k}\right)$. We also set to 1 the $i$ th bit of the edge-bitmaps of all black nodes visited. By Lemmas 1 and 2, all of this can be done in $O(\log n / \log \log n)$ time and we can also afford to add $\log \log n$ credits to each of the $O\left(\log n /(\log \log n)^{2}\right)$ new $i$-induced shortcuts.

We assumed that a node $v$ with edge $(v)[i]=1$ was encountered. If this is not the case, it means that $u$ should not be added to an existing tree in $\mathcal{F}_{i}$. Rather, a new tree should be formed consisting of a single edge $(u, p)$, where $p$ is the level $(i+1)$-ancestor of $u$ in $\mathcal{C}_{L}$. Clearly, the corresponding $i$-induced shortcuts can be added and edge-bitmaps updated within the same $O(\log n / \log \log n)$ time bound.

We can now conclude with the following theorem.

Theorem 1. There is a deterministic data structure for fully dynamic graph connectivity which supports edge insertions/deletions in $O\left(\log ^{2} n / \log \log n\right)$ amortized time per update and connectivity queries in $O(\log n / \log \log n)$ worst case time, where $n$ is the number of vertices of the graph. 


\section{Concluding Remarks}

We gave a deterministic data structure for fully-dynamic graph connectivity that achieves an amortized update time of $O\left(\log ^{2} n / \log \log n\right)$ and a worst-case query time of $O(\log n / \log \log n)$, where $n$ is the number of vertices of the graph. This improves the update time of Holm, de Lichtenberg, and Thorup [5] and Thorup [10] by a factor of $\log \log n$. We believe our improvement may extend to fully-dynamic minimum spanning tree, 2-edge, and/or biconnectivity.

There is still a small gap between upper and lower bounds. For instance, for $O(\log n / \log \log n)$ query time, [6] gives an $\Omega\left((\log n)^{1+\epsilon}\right)$ cell-probe lower bound for updates for constant $\epsilon>0$. Simultaneously getting $O(\log n)$ update and query time and improving the $O(\sqrt{n})$ worst-case update bound in [1] remain the main open problems for fully-dynamic graph connectivity.

\section{References}

[1] D. Eppstein, Z. Galil, G. F. Italiano, and A. Nissenzweig. Sparsification - a technique for speeding up dynamic graph algorithms. J. ACM, 44(5):669-696, 1997. See also FOCS'92.

[2] G. N. Frederickson. Data structures for on-line updating of minimum spanning trees, with applications. SIAM J. Comput., 14(4):781-798, 1985. See also STOC'83.

[3] M. R. Henzinger and V. King. Randomized dynamic graph algorithms with polylogarithmic time per operation. Proc. twenty-seventh annual ACM symposium on Theory of computing (STOC), 1995, pp. 519-527.

[4] M. R. Henzinger and M. Thorup. Sampling to provide or to bound: With applications to fully dynamic graph algorithms. Random Structures and Algorithms, 11(4):369-379, 1997. See also ICALP'96.

[5] J. Holm, K. de Lichtenberg, and M. Thorup. Poly-logarithmic deterministic fullydynamic algorithms for connectivity, minimum spanning tree, 2-edge, and biconnectivity. J. ACM, 48(4): 723-760, 2001. See also STOC'98.

[6] M. Pătraşcu and E. Demaine. Logarithmic Lower Bounds in the Cell-Probe Model. SIAM J. Comput., 35(4): 2006. Special issue 36th ACM Symposium on Theory of Computing (STOC 2004).

[7] D. Sleator and R. E. Tarjan. A data structure for dynamic trees. J. Comput. Syst. Sc., 26(3):362-391, 1983. See also STOC'81.

[8] R. E. Tarjan. Efficiency of a good but not linear set union algorithms. J. ACM, 22:215$225,1975$.

[9] M. Thorup. Decremental dynamic connectivity. Proc. 8th SODA, pp. 305-313, 1997.

[10] M. Thorup. Near-optimal fully-dynamic graph connectivity. Proc. thirty-second annual ACM symposium on Theory of computing (STOC), 2000, pp. 343-350. 